Published in "Physical Review E - Statistical,

Nonlinear, and Soft Matter Physics 84(2): 026101, 2011"

which should be cited to refer to this work.

\title{
Onset of cooperation between layered networks
}

\author{
Chang-Gui Gu, ${ }^{*}$ Sheng-Rong Zou, Xiu-Lian Xu, Yan-Qing Qu, Yu-Mei Jiang, and Da Ren He \\ College of Physics Science and Technology, Yangzhou University, Yangzhou 225002, P. R. China \\ Hong-Kun Liu \\ School of Statistics, South Western University of Finance and Economics, Chengdu 610074, P. R. China \\ Tao Zhou ${ }^{\dagger}$ \\ Web Sciences Center, University of Electronic Science and Technology of China, 610054 Chengdu, P. R. China, \\ Department of Modern Physics, University of Science and Technology of China, Hefei 230026, P. R. China, and \\ Department of Physics, University of Fribourg, Chemin du Musee 3, CH-1700 Fribourg, Switzerland
}

\begin{abstract}
Functionalities of a variety of complex systems involve cooperations among multiple components; for example, a transportation system provides convenient transfers among airlines, railways, roads, and shipping lines. A layered model with interacting networks can facilitate the description and analysis of such systems. In this paper we propose a model of traffic dynamics and reveal a transition at the onset of cooperation between layered networks. The cooperation strength, treated as an order parameter, changes from zero to positive at the transition point. Numerical results on artificial networks as well as two real networks, Chinese and European railway-airline transportation networks, agree well with our analysis.
\end{abstract}

\section{INTRODUCTION}

A complex system often contains different kinds of individuals and interactions, a system that is, sometimes, difficult and unnatural to be represented by a single network. For example, a transportation system involves airlines, railways, roads, and shipping lines, which is not easy to be fully described by an integrated network. An alternative is to separate the system into several subsystems, each of which can be respectively described by a network. Studies of such networks (e.g., air transportation networks [1,2], railway networks [3,4], networks of urban streets [5,6], etc.) provide insights into functions of subsystems; however, the interplay of subsystems is usually ignored, which may hinder the comprehensive understanding of the overall functions of the whole system.

Kurant et al. [7,8] observed that the load estimators based on physical networks cannot capture the real load of transportation systems, and thus they introduced a layered network model to describe and analyze transportation networks, where the lower layer is the physical infrastructure and the upper layer represents the traffic flows. This two-layer model has been further applied in analyzing a competitive network of household electrical appliance firms [9] and many others [10]. Zheng and Ergün [11] proposed a coevolution mechanism between two networks. Rodriguez [12] studied the coevolution of three networks, namely, the network of scientific journals, the coauthorship network of scientists, and the network of publications. These multinetwork models are supposed to give a more detailed and more accurate description of the objective systems. A few scientists started to concern themselves with

\footnotetext{
*Present address: Institute of Theoretical Physics and Department of Physics, East China Normal University, Shanghai 200062, P. R. China

${ }^{\dagger}$ zhutou@ustc.edu
}

the dynamics taking place on coupling networks, such as the spread of epidemics [13] and synchronization [14] between two networks. A breakthrough work is about cascading failure on layered networks [15], which has attracted increasing attention recently [16-18]. Thus far, it lacks investigations of traffic dynamics on layered transportation networks.

In this paper we propose a layered network model for transportation systems, where each layer represents a kind of transportation medium (e.g., airlines, railways, roads, etc.). This model is relevant yet different from Kurant's model [7,8], which emphasizes the different roles of physical and functional layers. Notice that people traveling from one place to another place usually utilize several media, which is here called cooperation between layered networks. We focus on a highly simplified traffic protocol between two layered networks, and reveal a transition at the onset of cooperation. The cooperation strength, treated as an order parameter, changes from zero to positive at the transition point. Numerical results on a Barabási-Albert (BA) model [19], Erdös-Rényi (ER) model [20], Newman-Watts (NW) model [21], and the real Chinese and European railway-airline transportation networks agree well with the analysis.

\section{MODEL}

For simplicity, we concentrate on a network consisting of two layers, and the methodology presented here can be easily extended to networks with several layers. A two-layer network, $L\left(G, G^{\prime}, E\right)$, consists of two layers (i.e., two subnetworks), $G$ and $G^{\prime}$, as well as links between two layers (called crossing links). Denote by $V$ and $V^{\prime}$ the sets of nodes of $G$ and $G^{\prime}$; in the layered network, different layers may contain the same nodes, and thus we denote by $V_{L}=V \cap V^{\prime}$ the joint set. We suppose that the links between two layers can connect only the same nodes but in different layers. For example, considering the Chinese railway-airline network with two layers, respectively, 


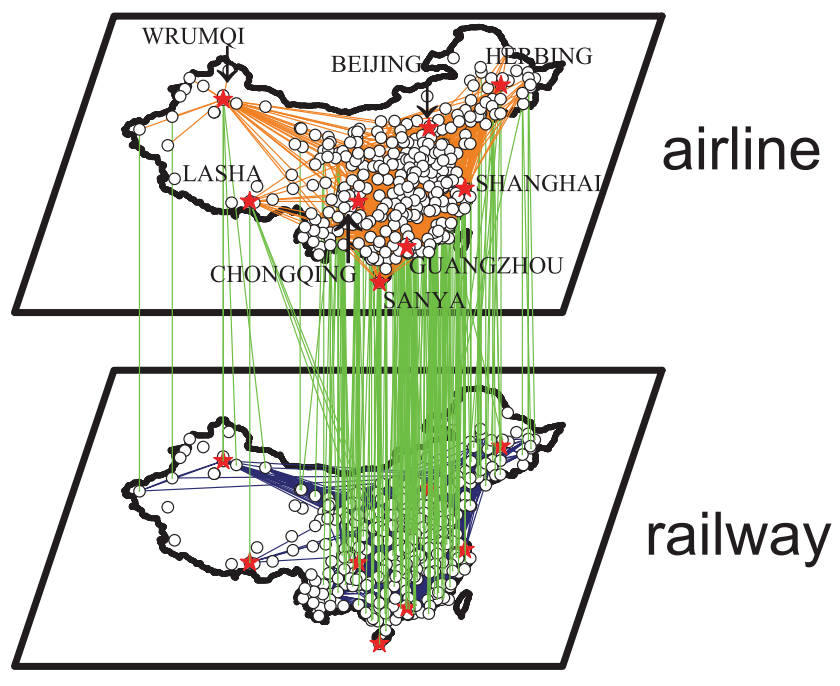

FIG. 1. (Color online) Illustration of the layered Chinese railwayairline network. Note that a few links are connecting two different nodes in different layers, which are ignored in the model.

representing railway and airline networks where each node denotes a city, a link connecting the same city in different layers represents a possibility that passengers can switch between the transportation media in this city (e.g., from the Shanghai Pudong Airport to the Shanghai Train Station by bus). Connections between different nodes in different layers are supposed to be rare and ignored in our model. Actually, if it happens frequently, we can add one more layer to present such kinds of connections and assume passing that through links adjacent to this new layer is of no cost (see below for the traffic model for the motivation of such assumption). Therefore, $E$ is essentially a subset of $V_{L}$. Figure 1 gives an illustration of the layered Chinese railway-airline networks.

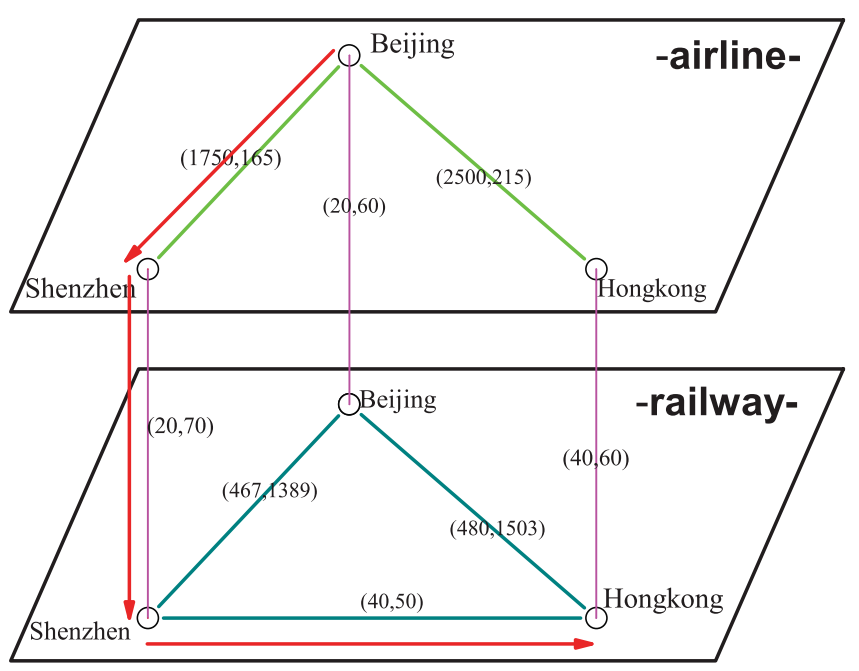

FIG. 2. (Color online) Illustration of a possible cooperation in a layered Chinese railway-airline network consisting of three big cities, Beijing, Shenzhen, and Hong Kong. The two numbers associated with a link denote the costs of money (RMB) and time (minutes) of the corresponding travel. Red path (labeled with arrows), from Beijing to Hong Kong, describes a possibly cooperated way costing 1810 RMB and 285 minutes.
Figure 2 illustrates a layered railway-airline network consisting of three big cities, Shenzhen, Beijing, and Hong Kong. Consider a passenger who would like to go from Beijing to Hong Kong: The fastest way is to fly directly to Hong Kong, taking 215 minutes but costing $2500 \mathrm{RMB}$, while the cheapest way is to take a train, costing only $480 \mathrm{RMB}$ but taking more than 25 hours. An alternative tradeoff may be to fly to Shenzhen first, and to take a bus from Shenzhen airport to Shenzhen train station, then to take a train to Hong Kong. This travel costs 1810 RMB and takes 285 minutes. We call a travel passing through one or more crossing links a cooperation between two layers. Motivated by the case illustrated in Fig. 2, we propose a traffic model on layered networks, where each link is assigned a certain weight accounting for the cost (e.g., price, time, or an integral of the two) to go through it. For simplicity, in the model, we assume two layers contain the same set of nodes, that is, $V=V^{\prime}$. If the starting point and destination are located in different layers, the cooperation happens in a trivial way, because the travel contains at least one crossing link. To exclude these trivial cooperations, we consider only the case where the starting point and destination both are located in the upper layer. We assume that every link in the upper layer is assigned a weight equal to 1 , and the weights of every crossing link and every link in the lower layer are $p$ and $q$, respectively.

Given the starting point and the destination, a passenger will follow the way with the smallest total weight, called the shortest weighted path. If there is more than one path with the shortest total weight (we have checked that this case happens rarely), the passenger will randomly choose one of them. Denote the numbers of upper-layer links, crossing links, and lower-layer links in such a shortest path connecting nodes $i$ and $j$ by $U_{i j}, C_{i j}$, and $L_{i j}$, respectively. To characterize the onset of cooperations, we define an order parameter called cooperation strength as $\Lambda=\frac{1}{N(N-1)} \sum_{i \neq j} \frac{C_{i j}}{U_{i j}+L_{i j}+C_{i j}}$, which equals zero if no cooperation occurs (i.e., $C_{i j}=0$ for any node pair $i$ and $j$ ) and is larger than zero when cooperations appear. The control parameter $d$ is defined as the difference between the link weights in upper and lower layers, say, $d=1-q$.

\section{RESULTS}

We start from an ideal case where the upper and lower layers have exactly the same topology. Consider a path connecting two upper-layer nodes that consist of $U$ links in the upper layer, $L$ links in the lower layer, and $C$ crossing links. This path will be chosen only if $U+C p+L q \leqslant U+L$, where the left part stands for the cost of this path, and the right part stands for the cost of a path involving only the upper-layer links (since the two layers are of exactly the same topology, these $U+L$ links can always form a path in the upper layer). Note that the minimum number of crossing links in an valid path is two, that is, at the transition point, $U+2 p+L q=U+L$. Substituting $q$ by $1-d$, one directly gets the transition point for the onset of cooperation, as $d_{c}=2 p / L$. Since each involved term has a linear contribution to the total sum, under the mean-field approximation, the average number of crossing links, $\langle C\rangle=\frac{1}{N(N-1)} C_{i j}$, should grow in a linear form with $\left(d-d_{c}\right)$. The cooperation strength $\Lambda$ is approximately 


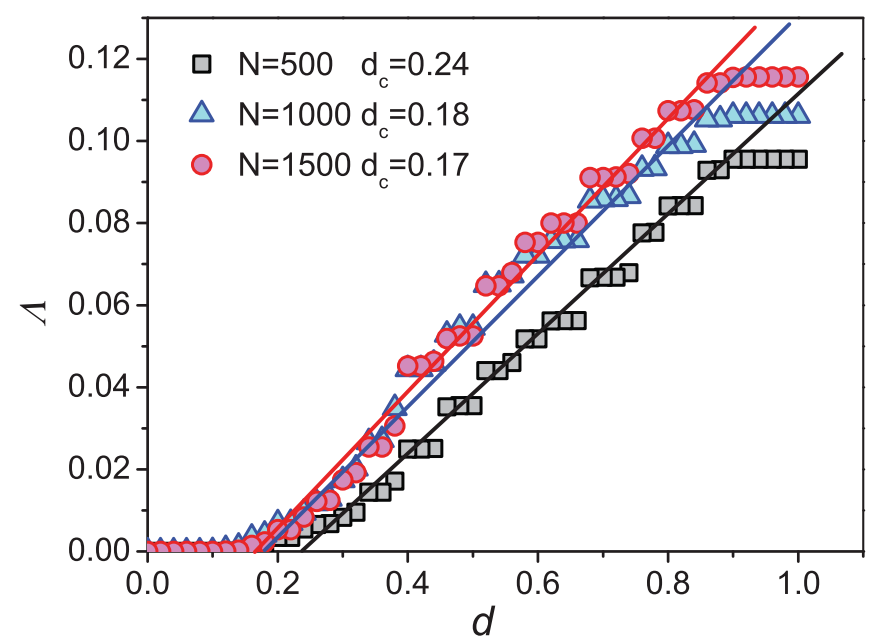

FIG. 3. (Color online) The cooperation strength $\Lambda$ as a function of $d$. The upper layer and lower layer have exactly the same topology, generated by the BA model [19]. Clearly, there are $N$ pairs of the same nodes in two layers, and we assign a crossing link to each of them with a probability 0.1 . The sizes of networks vary from 500 to 1500 , with a fixed average degree $\langle k\rangle=6$. The transition point $d_{c}$ is evaluated according to the intersection point of the fitting line and $x$ axis.

proportional to $\langle C\rangle$, and thus when $d$ exceeds the transition point, it grows approximately as $\Lambda \propto d-d_{c}$. Figure 3 reports the simulation results where the upper layer and lower layer have exactly the same topology, generated by the BA model [19]. The weight of a crossing link is set as the average weight of the links in the upper and lower layers, namely, $p=\frac{1+q}{2}$, and thus the transition point is $d_{c}=\frac{2}{2 L+1}$. Clearly,

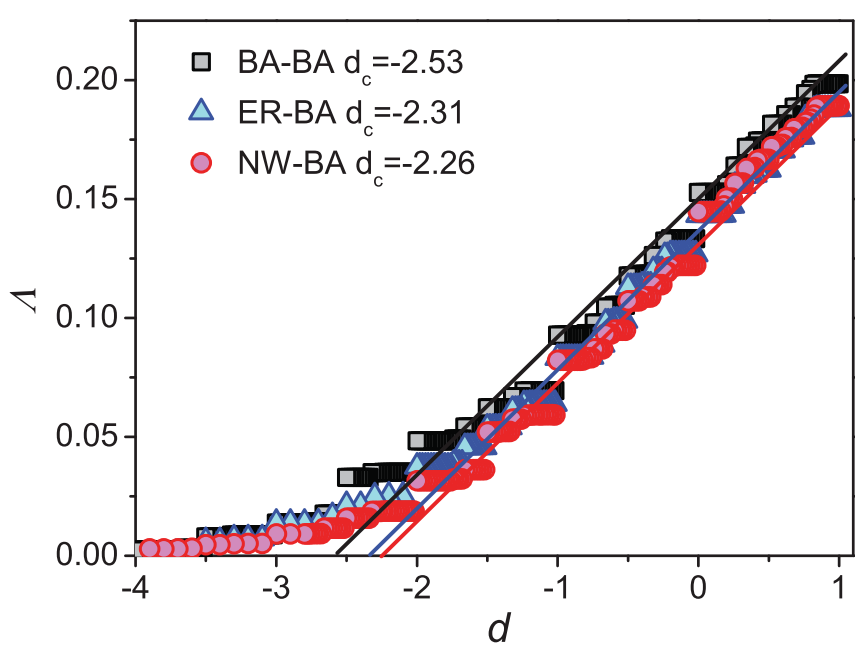

FIG. 4. (Color online) The cooperation strength $\Lambda$ as a function of $d$. The upper layers are always BA networks, and the lower layers are, respectively, an ER network, an NW network, and a BA network with a different topology from the upper layer. The size of every network is $N=1000$. The BA networks are of average degree $\langle k\rangle=6$, the connecting probability for ER networks is 0.01 , and for the NW network is 0.003 . For the NW network, the number of nearest neighbors is four. The nodes in upper and lower layers are randomly divided to be $N$ pairs, and a crossing link is attached to each pair with a probability 0.1 .

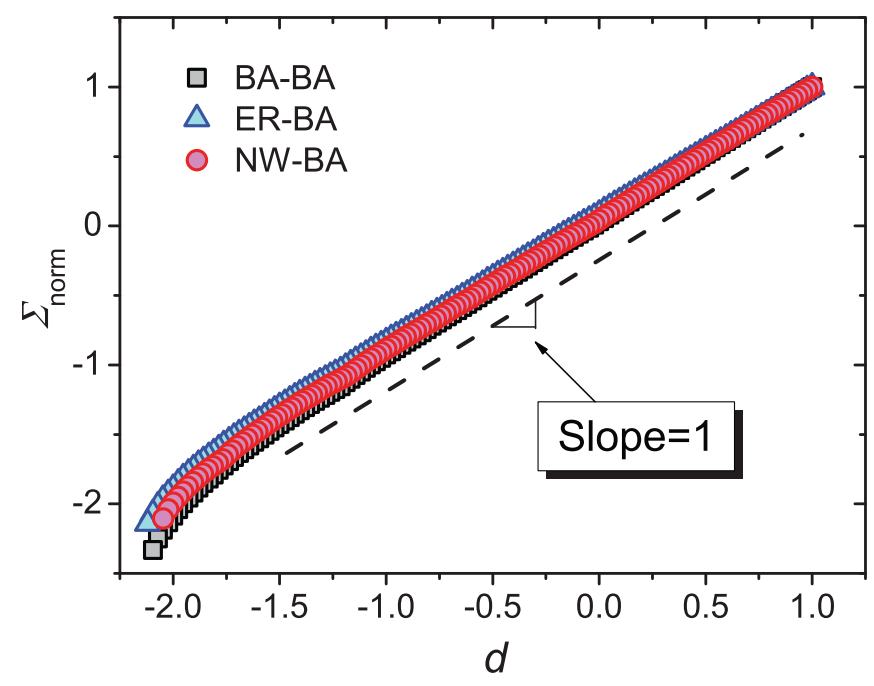

FIG. 5. (Color online) The normalized integral of order parameter as a function of $d$. The networks used for simulations and the parameters are the same as those of Fig. 4.

the cooperation tends to happen if the starting node $i$ and the destination $j$ are far away and there are crossing links adjacent to $i$ and $j$. In such a case, $L$ equals the shortest path length between $i$ and $j$. The perfect situation is that there are two crossing links adjacent to the two ends of a path with the longest length (i.e., the diameter). Denoting by $D$ the diameter of the upper-layer network (as the same as the lower-layer network), the transition point is about $d_{c}=\frac{2}{2 D+1}$. Note that this perfect situation does not hold every time since not all pairs of the same nodes located respectively in the upper and lower layers are connected by crossing links (for example, in Fig. 3, this probability is 0.1 ), and therefore the real transition point may be larger. For a BA network with $N$ varying from 500 to 1500 and $\langle k\rangle=6$, the typical diameter is 5 or 6 , leading to an approximate estimation of the transition points as $d_{c} \in(0.18,0.22)$, which agrees very well with the simulation results. It is also observed that the order parameter $\Lambda$ grows in a linear form when $d$ exceeds the transition point, in accordance with our expectation. In addition, as the network size increases, the diameter becomes larger and transition point becomes smaller, which is also in accordance with the simulations.

Clearly, the situation will be different if the upper and lower layers have different topologies. That is, the crossing links can provide some shortcuts, and thus even when $d<0$, cooperation may occur. For example, there is no direct train from Wrumqi to Sanya, but by moving from the train station to the airport of Wrumqi (i.e., a crossing link), one can quickly

TABLE I. Basic information of the empirical data sets used in Fig. 6, where $N$ and $M$ denote the number of nodes and edges, respectively.

\begin{tabular}{lccc}
\hline \hline Networks & $N$ & $M$ & References \\
\hline Chinese railway & 3036 & 275616 & {$[22,23]$} \\
Chinese airline & 122 & 1467 & {$[24,25]$} \\
European railway & 362 & 1345 & {$[26]$} \\
European airline & 127 & 413 & {$[27]$} \\
\hline \hline
\end{tabular}




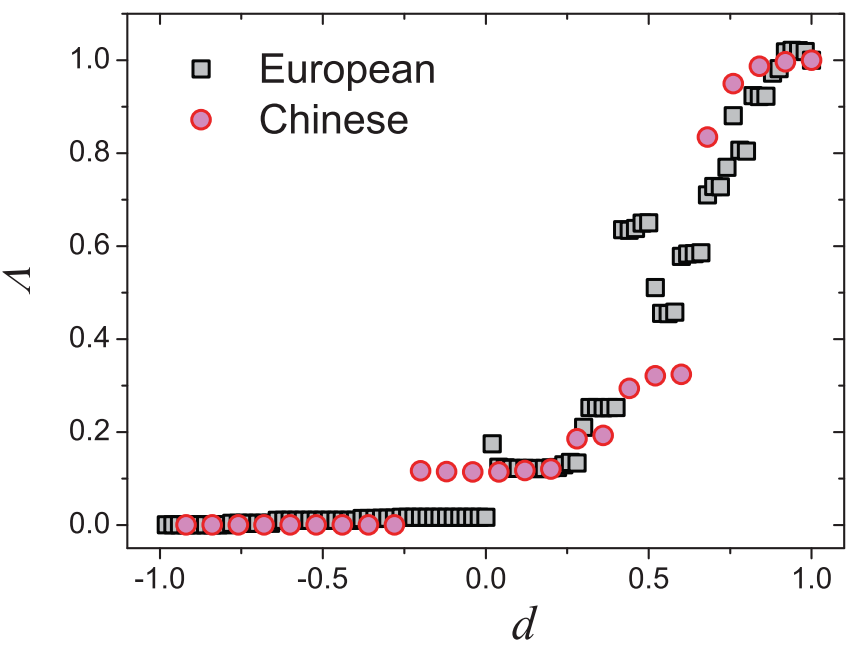

FIG. 6. (Color online) The cooperation strength $\Lambda$ as a function of $d$. The upper and lower layers are railway and airline networks. The black squares and red circles denote the results for European and Chinese transportation systems, respectively.

arrive at Sanya through a direct airline. Figure 4 reports the simulation results where the upper layers are always BA networks and the lower layers are, respectively, an ER network, an NW network, and a BA network with a different topology from the upper layer. In accordance with our expectation, when the upper and lower layers have different topologies, the cooperation happens for $d<0$. Even when the upper and lower layers have different topologies, the cooperation pattern may be governed by the same regularities. An ansatz is that $\Lambda(d)=A\left(d-d_{c}\right)+B$ for $d \geqslant d_{c}$ with $A$ and $B$ two constants. When $d=d_{c}, \Lambda=0, B$ must be zero. Therefore, the order parameter scales linearly with the difference from transition point as $\Lambda(d)=A\left(d-d_{c}\right)$. The integral of $\Lambda$ from $d_{c}$ to $d$ is $\Sigma(d)=\int_{d_{c}}^{d} \Lambda(x) d x=\frac{A}{2}\left(d-d_{c}\right)^{2}$. Since $d=1-q \leqslant 1$, the maximum value of the integral is $\Sigma_{\max }=$ $\int_{d_{c}}^{1} \Lambda(x) d x=\frac{A}{2}\left(1-d_{c}\right)^{2}$. The normalized integral is defined as $\Sigma_{\text {norm }}(d)=\left(1-d_{c}\right) \sqrt{\frac{\Sigma(d)}{\Sigma_{\max }}}+d_{c}=d$. That is to say, if our analysis is valid, for different cases in Fig. 4, the curves $\Sigma_{\text {norm }}(d)$ should collapse to a master curve lying along the diagonal line. As shown in Fig. 5, the three curves presented in Fig. 4 have collapsed nicely into a master curve with slope 1 , which agrees well with our inference.

To see the relevance of the present model, we next investigate two real layered networks: One is the coupling of the Chinese railway network and the Chinese airline network, and the other is the coupling of the European railway and airline networks. The basic information about these networks is presented in Table I. Figure 6 reports the simulation results for these two real networks. Notice that, in the real case, the upper and lower layers contain a widely different number of nodes, and many nodes appear only in one layer. In addition, the costs of different flights or trains, estimated by either time or price (the real selection of human beings are even more complicated), should be different, which is against the assumption of identical weight. Therefore, the results cannot be quantitatively predicted by the theoretical model. However, compared with the results of the model shown in Fig. 4, the empirical results agree with the model qualitatively; that is to say, the cooperation happens even with negative $d$ (but with very small strength), and the strength will soon grow in an approximately linear way.

\section{CONCLUSION}

A layered representation of interacting networks can facilitate the description and analysis of many systems. In some of them, networks in different layers depend on one another, and in some other systems, different layers cooperate to accomplish some certain functionalities. A transportation system is a typical example of the latter cases, in which long-distance travel usually involves an airline, railway, route, etc. Here we proposed a model of layered transportation networks and revealed a transition at the onset of cooperation. The cooperation strength, treated as an order parameter, changes from zero to positive at the transition point. Our analysis may shed some light on the understanding of coupling networks.

\section{ACKNOWLEDGMENTS}

This work is supported by the National Natural Science Foundation of China under Grant Nos. 10635040 and 70671089 and the National Basic Research Program of China (973 Program No. 2006CB705500).
[1] A. Barrat, M. Barthélemy, R. Pastor-Satorras, and A. Vespignani, Proc. Natl. Acad. Sci. USA 101, 3747 (2004).

[2] R. Guimerà, S. Mossa, A. Turtschi, and L. A. N. Amaral, Proc. Natl. Acad. Sci. USA 102, 7794 (2005).

[3] P. Sen, S. Dasgupta, A. Chatterjee, P. A. Sreeram, G. Mukherjee, and S. S. Manna, Phys. Rev. E 67, 036106 (2003).

[4] K. A. Seaton and L. M. Hackett, Physica A 339, 635 (2004).

[5] A. Cardillo, S. Scellato, V. Latora, and S. Porta, Phys. Rev. E 73, 066107 (2006).

[6] M. Barthélemy and A. Flammini, Phys. Rev. Lett. 100, 138702 (2008).

[7] M. Kurant and P. Thiran, Phys. Rev. Lett. 96, 138701 (2006).
[8] M. Kurant, P. Thiran, and P. Hagmann, Phys. Rev. E 76, 026103 (2007).

[9] J.-M. Yang, W.-J. Wang, and G.-R. Chen, Physica A 388, 2435 (2009).

[10] X.-L. Xu, Y.-Q. Qu, S. Guan, Y.-M. Jiang, and D.-R. He, Europhys. Lett. 93, 68002 (2011).

[11] D. Zheng and G. Ergün, Adv. Complex Syst. 6, 507 (2003).

[12] M. A. Rodriguez, Int. J. Public Inf. Sys. 1, 13 (2007).

[13] M. E. J. Newman, Phys. Rev. Lett. 95, 108701 (2005).

[14] C. Li, W. Sun, and J. Kurths, Phys. Rev. E 76, 046204 (2007).

[15] S. V. Buldyrev, R. Parshani, G. Paul, H. E. Stanley, and S. Havlin, Nature (London) 464, 1025 (2010). 
[16] R. Parshani, S. V. Buldyrev, and S. Havlin, Phys. Rev. Lett. 105, 048701 (2010).

[17] R. Parshani, C. Rozenblat, D. Ietri, C. Ducruet, and S. Havlin, Europhys. Lett. 92, 68002 (2010).

[18] J. Shao, S. V. Buldyrev, S. Havlin, and H. E. Stanley, Phys. Rev. E 83, 036116 (2011).

[19] A.-L. Barabási and R. Albert, Science 286, 509 (1999).

[20] P. Erdös and A. Rényi, Publ. Math. Inst. Hung. Acad. Sci. 5, 17 (1960).

[21] M. E. J. Newman and D. J. Watts, Phys. Lett. A 263, 341 (1999).
[22] Y.-L. Wang, T. Zhou, J.-J. Shi, J. Wang, and D.-R. He, Physica A 388, 2949 (2009).

[23] S.-R. Zou, T. Zhou, A.-F. Liu, X.-L. Xu, and D.-R. He, Phys. Lett. A 374, 4406 (2010).

[24] H.-K. Liu and T. Zhou, Acta Phys. Sin. 56, 106 (2007).

[25] H.-K. Liu, X.-L. Zhang, and T. Zhou, Physics Procedia 3, 1781 (2010).

[26] [http://www.eurail.com/eurail-timetables/].

[27] [http://www.europebyair.com/efp/index.jsp/]. 